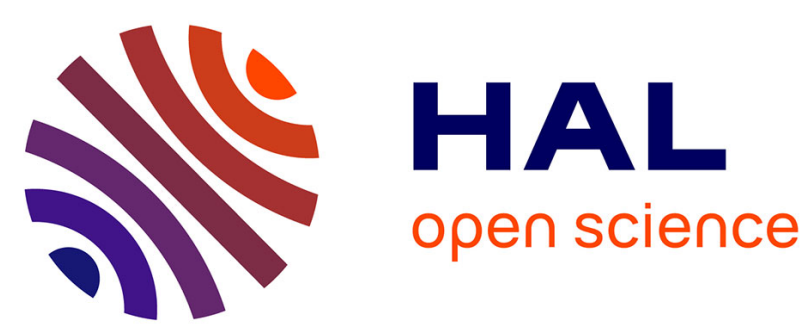

\title{
Pollution et stress oxydant
}

B. Geny, A.-L. Charles, A. Lejay, A. Meyer

\section{To cite this version:}

B. Geny, A.-L. Charles, A. Lejay, A. Meyer. Pollution et stress oxydant. Revue francaise d'allergologie, 2019, 59, pp.174 - 176. 10.1016/j.reval.2019.02.195 . hal-03486363

\section{HAL Id: hal-03486363 https://hal.science/hal-03486363}

Submitted on 20 Dec 2021

HAL is a multi-disciplinary open access archive for the deposit and dissemination of scientific research documents, whether they are published or not. The documents may come from teaching and research institutions in France or abroad, or from public or private research centers.
L'archive ouverte pluridisciplinaire HAL, est destinée au dépôt et à la diffusion de documents scientifiques de niveau recherche, publiés ou non, émanant des établissements d'enseignement et de recherche français ou étrangers, des laboratoires publics ou privés.

\section{다(1) $\$$}

Distributed under a Creative Commons Attribution - NonCommercial| 4.0 International 


\section{Pollution et stress oxydant}

\section{Pollution and oxidative stress}

*Bernard Geny ${ }^{1,2}$, Anne-Laure Charles ${ }^{1}$, Anne Lejay ${ }^{1,2,3}$, Alain Meyer ${ }^{1,2}$

1. Equipe d'Accueil 3072, Mitochondrie, Stress oxydant et Protection Musculaire, Fédération de Médecine Translationelle de Strasbourg, Université de Strasbourg, Institut de Physiologie, 67000 Cedex, France.

2. Service de Physiologie et d'Explorations Fonctionnelles, Pôle de Pathologie Thoracique Hôpitaux Universitaires, CHRU de Strasbourg, 67000 Cedex, France.

3. Service de chirurgie vasculaire et transplantation rénale, Pôle de cardiologie, Hôpitaux Universitaires, CHRU de Strasbourg, 67000 Cedex, France.

Auteur correspondant / Corresponding author : Prof. Bernard Geny, Hôpitaux Universitaires de Strasbourg, Nouvel Hôpital Civil, Service de Physiologie et d'Explorations Fonctionnelles

1 place de l'Hôpital

67091 Strasbourg Cedex, France

Mail: bernard.geny@chru-strasbourg.fr

Téléphone / phone : +33 369550660 or 0369550879

Fax : +33 369551826 
Mots-clés : pollution; stress oxydant; mitochondrie

Keywords: pollution; oxidative stress; mitochondria

La pollution peut se définir comme la dégradation d'un écosystème par introduction, souvent humaine, de substances ou de radiations chimique, nucléaire, sonore et/ou lumineuse qui peuvent toucher préférentiellement les sols, l'eau et/ou l'air et bien sûr, les êtres vivants. Nous parlerons ici essentiellement de pollution de l'air, mélange complexe d'éléments chimiques, biologiques et physiques (gaz, liquides semi volatiles et particules) en constante évolution, dont l'impact varie selon des paramètres individuels (âge, pathologies préexistantes) et généraux (géographie, météorologie, activités humaines). Les polluants sont extérieurs et intérieurs et on n'insiste probablement pas assez sur les effets délétères de la pollution intérieure qui semble responsable d'environ la moitié des 7 millions de morts prématurées observées annuellement dans le monde.

La pollution extérieure est d'origine naturelle (érosion des sols, éruption volcanique, incendie) ou humaine (industrie, transport, agriculture, chauffage). Parmi les polluants les plus néfastes pour la santé, notons les particules, classées cancérigènes, et dont les effets sur la mortalité et la morbidité cardio-respiratoire sont bien documentés. Les données concernent essentiellement les particules fines, PM 10 et 2,5 $\mu \mathrm{m}$, mais les particules ultra fines sont probablement plus toxiques. L'ozone et le dioxyde d'azote (NO2) etc. participent aux atteintes observées chez l'Homme. La pollution intérieure est aussi importante car nous passons plus de $80 \%$ de notre temps dans des lieux fermés (domicile, travail, école, transports). Le tabac est le premier polluant intérieur suivi des produits d'entretien (ménagers, parfums d'intérieur, encens), de bricolage (peintures, colles, solvants) et des produits utilisés pour la fabrication des meubles (formaldéhyde). Arrêtez de fumer!

Les études épidémiologiques montrent une association certaine entre les pathologies respiratoires et cardio-vasculaires et la pollution de l'air [1-5]. Classiquement, une exposition aigüe (quelques heures à quelques jours) peut entraîner de simples irritations (oculaire, des voies respiratoires) voire des crises d'asthme et des décompensations cardiovasculaire et respiratoire, pouvant conduire à l'hospitalisation ou au décès. Une exposition chronique (plusieurs années), contribue au développement ou à l'aggravation de maladies chroniques telles que les cancers (poumon), les pathologies cardiovasculaires (dysfonction endothéliale, HTA, infarctus du myocarde, AVC), respiratoires (BPCO, asthme, pathologies interstitielles), neurologiques et auto-immunes (démences, Parkinson, lupus, diabète). En France, l'impact sanitaire prépondérant est dû à une exposition au long cours, à des niveaux moyens de pollution. La part des effets sanitaires attribuables aux pics de pollution semble faible.

L'atteinte respiratoire conjugue altération des voies respiratoires et des cellules de l'immunité, qui favorisent les réactions allergiques [1]. Des polluants inhalés peuvent avoir des effets cardiovasculaires et systémiques en activant des cellules inflammatoires pulmonaires qui relâchent des médiateurs de l'inflammation (cytokines), en activant des récepteurs alvéolaires qui stimulent des afférents nerveux modulant le système autonome et, en franchissant directement la barrière alvéolo capillaire et passer ainsi dans la circulation [3]. Ainsi, les particules ultrafines se retrouvent facilement dans la circulation, les cellules et même dans les mitochondries.

Parmi les principaux mécanismes impliqués, le stress oxydant et les dysfonctions mitochondriales [2-5] semblent jouer un rôle important. Le stress oxydant est un état de déséquilibre entre la production d'espèces réactives (radicaux libres) de l'oxygène (RLO) et le 
système anti oxydant endogène. Un stress oxydant peut donc être dû à une surproduction de RLO et/ou à une diminution de l'activité anti oxydante.

Un radical libre est une molécule instable très réactive qui présente un électron non apparié, et qui n'a de cesse de vouloir arracher un électron à la molécule voisine. Des RLO importants sont l'anion superoxide $(\bullet \mathrm{O} 2-)$, le peroxyde d'hydrogène, les radicaux hydroxyles. A côté de certaines enzymes (xanthine oxydase, NADPH oxydase), la mitochondrie est la source principale de RLO. En effet, si $98 \%$ de l'oxygène utilisé par la mitochondrie lui permet de générer l'indispensable ATP, les $2 \%$ restant favorisent la production de RLO. En faible quantité, ces radicaux libres ne sont pas délétères. Par contre, en quantité importante et/ou si l'exposition est prolongée, ces RLO entraînent des altérations majeures incluant peroxydation lipidique, carboxylation protéique et/ou fragmentation de l'ADN, voire mort cellulaire par apoptose secondaire à l'ouverture du pore de transition mitochondrial [6, Figure]. Toutes les cellules et ainsi tous les organes peuvent être touchés, en fonction de leurs capacités anti oxydantes intrinsèques. Ainsi, si le stress oxydant n'est pas considéré comme une maladie en soit, c'est un mécanisme physiopathologique à l'origine de très nombreuses pathologies aigües et chroniques.

Un anti oxydant est toute substance qui, à une concentration faible par rapport à substrat oxydable, retarde ou empêche l'oxydation de ce substrat. Nous possédons des défenses antioxydantes enzymatiques (superoxide dismutase (SOD), catalase, glutathion peroxidase) ou non (glutathion, vitamines) qui nous protègent le plus souvent efficacement.

Le lien entre pollution et stress oxydant est maintenant bien étayé. De nombreuses études ont montré que l'exposition à une pollution de l'air augmente les marqueurs de stress oxydant et ses atteintes cellulaires caractéristiques. L'inflammation participe à ces lésions.

En plus de réduire les sources de pollution, il faut continuer d'informer la population des risques (sensibilité accrue des enfants, personnes âgées et des patients) et utiliser les traitements habituels des pathologies cardio-respiratoires.

Le rôle du stress oxydant est majeur, c'est un médiateur clé qui institue et exacerbe le processus pathologique. Fait intéressant, si l'intérêt pharmacologique ou alimentaire d'une modulation du stress oxydant reste à préciser, quelques données suggèrent que l'emploi d'antioxydants peut réduire les effets délétères de la pollution. Plus urticant; puisqu'une faible exposition aux RLO induit une biogenèse mitochondriale et une augmentation des défenses anti oxydantes intrinsèques, on pourrait considérer le cyclisme urbain comme un préconditionnement «pollutif » qui protégerait contre un stress oxydant majeur ultérieur. 
1. Li N, Georas S, Alexis N, Fritz P, Xia T, Williams MA,et al. A work group report on ultrafine particles (American Academy of Allergy, Asthma \& Immunology): Why ambient ultrafine and engineered nanoparticles should receive special attention for possible adverse health outcomes in human subjects. J Allergy Clin Immunol. 2016;138:386-396.

2. Weichenthal SA, Lavigne E, Evans GJ, Godri Pollitt KJ, Burnett RT. Fine Particulate Matter and Emergency Room Visits for Respiratory Illness. Effect Modification by Oxidative Potential. Am J Respir Crit Care Med. 2016;194:577586.

3. Niemann B, Rohrbach S, Miller MR, Newby DE, Fuster V, Kovacic JC. Oxidative Stress and Cardiovascular Risk: Obesity, Diabetes, Smoking, and Pollution: Part 3 of a 3-Part Series. J Am Coll Cardiol. 2017 Jul 11;70(2):230-251.

4. Boovarahan SR, Kurian GA. Mitochondrial dysfunction: a key player in the pathogenesis of cardiovascular diseases linked to air pollution. Rev Environ Health. 2018 Jun 27;33(2):111-122.

5. Valavanidis A, Vlachogianni T, Fiotakis K, Loridas S. Pulmonary oxidative stress, inflammation and cancer: respirable particulate matter, fibrous dusts and ozone as major causes of lung carcinogenesis through reactive oxygen species mechanisms. Int J Environ Res Public Health. 2013 Aug 27;10(9):3886-907.

6. Lejay A, Meyer A, Schlagowski AI, Charles AL, Singh F, Bouitbir J, et al. Mitochondria: mitochondrial participation in ischemia-reperfusion injury in skeletal muscle. Int J Biochem Cell Biol. 2014;50:101-105. 
Figure : Interactions entre la pollution, le stress oxydant et les mitochondries.

\section{Pollution}

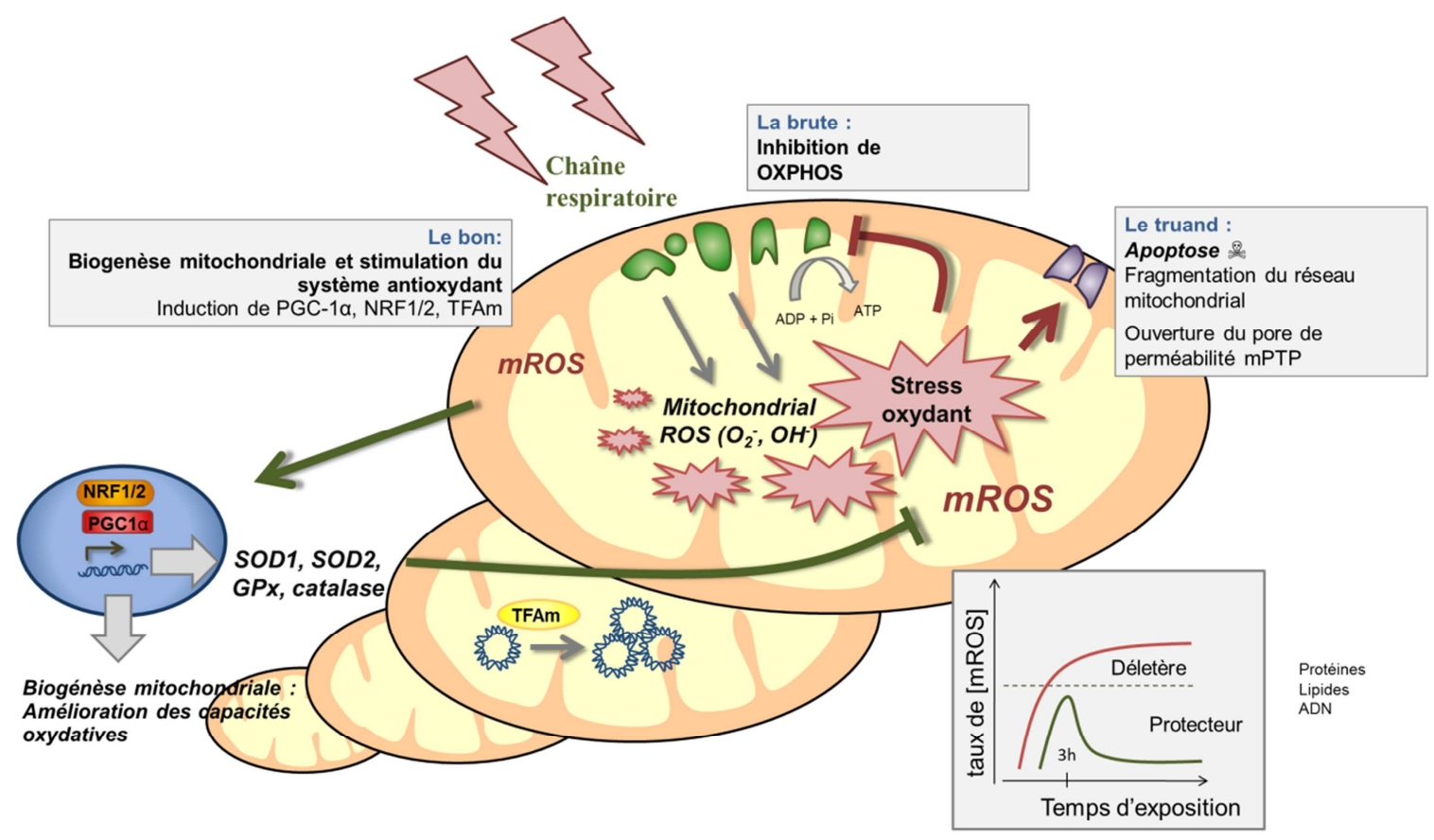

